\title{
Avaliação da contaminação parasitológica de rúculas (Eruca sativa) comercializadas em Erechim, RS, Brasil
}

\author{
Parasitological contamination evaluation of arugula \\ (Eruca sativa) marketed in Erechim-RS, Brazil
}

Recebido em: 08/08/2014

Aceito em: 11/09/2014
Maiara Carla MATIASSO;

Itamar Luís GONÇALVES; Neiva Aparecida GRAZZIOTIN Universidade Regional Integrada do Alto Uruguai e das Missões URI. Avenida Sete de Setembro, 1621, Erechim, RS, Brasil. Cx.Postal 743.Cep99700-000.E-mail:neivagra@uri.com.br

\section{ABSTRACT}

This study aimed to evaluate the presence of enteroparasites in arugula (Eruca sativa) commercialized in Erechim, RS. In the period from March to May 2013 were analyzed, through spontaneous sedimentation method, 40 samples, obtained from markets. Among the argulas analysed, 22.5 showed contamination with trophozoites and/or cysts of amoeba. Eggs or larvae of pathogenic helminths were not found in the analysed samples. Vegetables consumed without previous cooking can present standard of sanitary and hygienic quality inadequate, condition that emphasizes the need for greater guidance to producers and handlers, with regard to its correct handling and hygiene.

Keywords: Eruca sativa; amoeba; intestinal parasites

\section{RESUMO}

Este estudo teve como objetivo avaliar a contaminação por enteroparasitas em amostras de rúcula (Eruca sativa) comercializadas na cidade de Erechim-RS. No período de março a maio de 2013, foram analisadas, pelo método de sedimentação espontânea, 40 amostras, obtidas em mercados e feiras do produtor. Dentre as rúculas analisadas, 22,5\% apresentaram contaminação por trofozoítos e/ou cistos de ameba. Não foram encontrados ovos ou larvas de helmintos patogênicos ao homem. Vegetais consumidos sem cozimento prévio podem apresentar padrão de qualidade higiênicosanitária inadequado, o que destaca a necessidade de maior orientação aos produtores e manipuladores, no que se refere à sua correta manipulação e higienização.

Palavras Chave: Eruca sativa; amebas; parasitas intestinais 


\section{INTRODUÇÃO}

As doenças parasitárias são mundialmente frequentes, apresentando maior prevalência nos países em desenvolvimento (1). Na América Latina e no Brasil, este grupo de infecções constitui um importante problema de saúde pública $(2,3)$. Condições incipientes de saneamento básico consistem em um fator determinante na cadeia de transmissão das enteroparasitoses (4). Nesse contexto, a água e os alimentos atuam como veículo de transmissão de organismos prejudiciais à saúde $(5,6)$ e a falta de higiene na manipulação de alimentos pode representar uma fonte de disseminação de enteroparasitas $(4,7)$.

$\mathrm{O}$ consumo de vegetais in natura exerce papel fundamental em qualquer dieta, em função de seu alto conteúdo de vitaminas, fibras alimentares e baixo teor calórico (8). Entretanto, quando estes são higienizados inadequadamente, podem transmitir doenças causadas por microrganismos e/ou parasitas intestinais (9). Soma-se ao contexto elevados índices de contaminação por helmintos e protozoários encontrados nestes alimentos $(10,11)$.

Cistos de Entamoeba histolytica, protozoário responsável pela amebíase, podem estar presentes em vegetais in natura concomitantemente com cistos e/ou trofozoítos de amebas de vida livre. Estes organismos constituem um grupo de protozoários presentes no solo e em pequenos reservatórios de água fresca (12). Algumas espécies de amebas de vida livre podem comportar-se como parasitas facultativos em seres humanos e animais domésticos, sendo capazes de invadir o sistema nervoso central e outros tecidos $(13,14)$.

Em função do consumo in natura de rúcula (Eruca sativa), o estudo teve como objetivo investigar a presença de estruturas parasitárias de helmintos e protozoários nesse alimento comercializado em feiras e supermercados de Erechim, RS (Figura 1).

Figura 1: Localização da cidade onde foram obtidas as amostras.

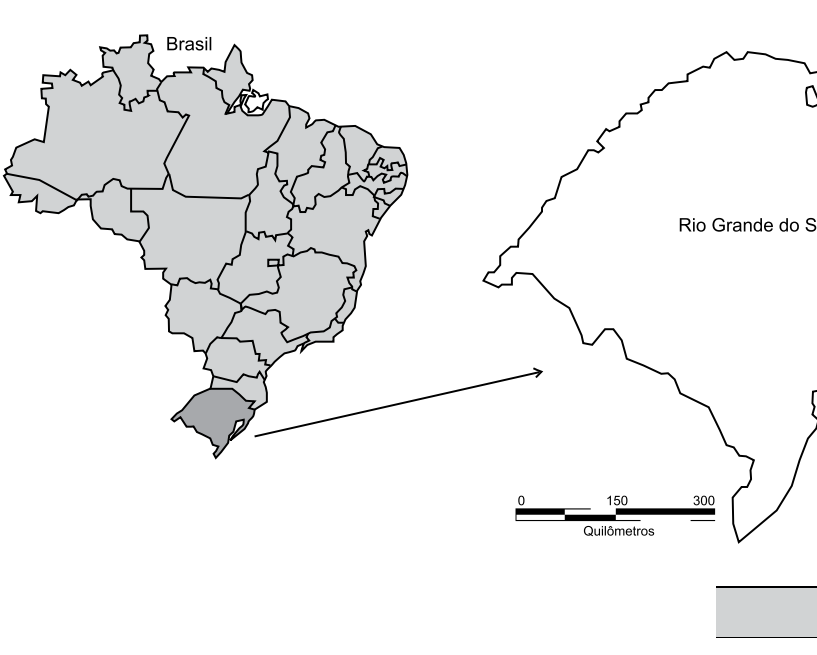

Fonte: Lageplan URI Erechim.

\section{MATERIAL E MÉTODOS}

Foram adquiridas 40 amostras de rúcula, sendo 20 em feiras livres e 20 em supermercados de Erechim, RS (Figura 1), entre os meses de março e maio de 2013. As amostras foram acondicionadas individualmente em sacos plásticos e os mesmos foram lacrados e transportados ao laboratório de Parasitologia da Universidade Regional Integrada do Alto Uruguai e das Missões - URI
Erechim. A unidade amostral foi constituída pelo maço de rúcula, formado por ramos e folhas agrupados por um laço. Para a pesquisa de enteroparasitas nas amostras, foram adicionados $300 \mathrm{~mL}$ de água destilada e três gotas de detergente neutro em cada amostra acondicionada em saco plástico. Após agitação, o conteúdo foi filtrado em cálice descartável com peneira para exames parasitológicos $(6,15)$. Decorridas 24 horas de repouso, foram preparadas duas lâminas a partir do sedimento, as quais 
foram analisadas ao microscópio óptico em objetiva de 10x e 40 x para identificação e confirmação das estruturas parasitárias.

\section{RESULTADOS E DISCUSSÃO}

Os resultados da avaliação parasitológica das 40 amostras de rúculas (Eruca sativa) estão descritos na Tabela 1. Em relação à totalidade das amostras 22,5\% (9/40) apresentaram contaminação por trofozoítos e/ou cistos de ameba, sendo $20 \%$ (4/20) das amostras provenientes de feiras do produtor e $25 \%$ (5/20) de supermercados. Todas as amostras apresentaram larvas de vida livre e protozoários ciliados e/ou flagelados. Porém, ovos ou larvas de helmintos patogênicos ao homem não estiveram presentes nas amostras analisadas.

As parasitoses intestinais estão entre as doenças mais frequentes do mundo (16), podendo afetar a produtividade, a capacidade física e mental em diferentes faixas etárias (17). Entre as enteroparasitoses prevalentes no Brasil, a amebíase, doença causada pela Entamoeba histolytica, pode causar lesões e sintomas intestinais e extra-intestinais, e seu contágio está associado com a ingestão de cistos presentes na água e alimentos inadequadamente higienizados $(18,19)$.

Ecossistemas aquáticos receptores de esgotos, localizados próximos de áreas urbanas permitem a existência de matéria orgânica e bactérias que servem de alimento para protozoários (20). Esta condição concomitantemente ao uso extensivo de águas fluviais para irrigação pode estar associada com o alto percentual de protozoários de vida livre encontrados em hortaliças.

A presença de amebas de vida livre não se encontra restrita a alimentos. Foram identificadas as amebas de vida livre potencialmente patogênicas Naegleria fowleri e Acanthamoeba spp. em poeira de ambiente hospitalar no Brasil $(21,22)$, e em águas com esgoto industrial na Áustria (23). Em um estudo realizado na Espanha durante um ano em 223 amostras de água, utilizando RT-PCR e cultura, foi identificada a presença de Acanthamoeba spp. em $99,1 \%$ das amostras analisadas (24).

Tabela 1 - Análise parasitológica de Eruca sativa comercializadas em supermercado e feira do produtor em Erechim, RS, no período de março a maio de 2013

\begin{tabular}{|l|c|c|c|c|c|c|}
\hline \multirow{2}{*}{ Parasita } & \multicolumn{3}{|c|}{ Feira } & Supermercado & \multicolumn{3}{c|}{ Total } \\
\cline { 2 - 7 } & $\mathrm{n}$ & $\%$ & $\mathrm{n}$ & $\%$ & $\mathrm{n}$ & $\%$ \\
\hline Trofozoítos e/ou cistos de ameba & 4 & 20 & 5 & 25 & 9 & 22,5 \\
\hline Larvas de vida livre & 20 & 100 & 20 & 100 & 40 & 100 \\
\hline Protozoários ciliados e/ou flagelados & 20 & 100 & 20 & 100 & 40 & 100 \\
\hline
\end{tabular}

Encefalite amebiana primária produzida por Naegleria fowleri tem sido reportada em humanos (13) e animais rurais (14). O agente etiológico apresenta ampla distribuição (25), e contaminações humanas têm ocorrido em águas recreacionais (26). Espécies de Acanthamoeba podem produzir infecções crônicas na córnea (27).

Além do potencial de produzir infecções, amebas de vida livre podem atuar como reservatórios para bactérias patogênicas, capazes de resistir à fagocitose $(28,29)$. No interior de amebas de vida livre podem sobreviver Legionella pneumophila, Acinetobacter baumannii, Pseudomonas aeruginosa e Stenotrophomonas maltophilia, associados com infecções nosocomiais (29).

Diversos trabalhos reportam aspectos relacionados à inadequada higienização de hortaliças consumidas cruas, evidenciando elevados índices de contaminação parasitária em rúcula (11,30-33).

Em relação à contaminação de rúcula por ovos e/ ou larvas de enteroparasitas, resultados diferentes aos do presente estudo foram obtidos na análise de 30 amostras de rúcula comercializada em Maringá, que constatou índices de parasitismo superiores a $80 \%$, sendo encontrados com mais elevada frequência ovos e larvas das superfamílias Rhabdiasoidea e Ancylostomatoidea, em $30 \%$ das amostras (11).

Outro estudo efetuado em Jataí-GO constatou a prevalência de ovos de ancilostomídeos, Toxocara spp. e Ascaris sp., em 55\% das amostras de rúcula analisadas (30). De forma semelhante ovos e larvas das famílias Ancylostomatoidea e de Rhabdiasoidea foram as formas parasitárias prevalentes em análise realizada em feiras livres de Maringá. Neste estudo foi encontrado 33\% de contaminação em rúculas em amostras coletadas entre 2002/2003. Índices mais elevados de parasitismo (78\%) são descritos em rúculas provenientes da mesma região entre o período de 2003/2004 (31).

Um amplo número de protozoários e helmintos podem contaminar amostras de rúcula. Resultados diferen- 
tes aos encontrados no presente estudo são descritos em Nova Xantina, MT, onde foram identificados dez diferentes parasitas em amostras de rúcula, sendo os principais, Ancylostoma spp. Ascaris lumbricoides, Entamoeba coli, Schistosoma mansoni e Giardia lamblia (32).

Índices de parasitismo por Entamoeba spp. mais elevados em relação ao presente trabalho foram descritos em investigação realizada em Florianópolis, SC, onde a análise de 250 amostras de rúcula evidenciou $74,4 \%$ de contaminação por Entamoeba spp. (33).

Dessa forma, a segurança alimentar está diretamente relacionada com a correta higienização dos alimentos e de indivíduos que executam atividades relacionadas com seu processamento e preparo. Segundo a Resolução ${ }^{\circ}$ 216/2004 da Agência Nacional de Vigilância Sanitária, Anvisa, a correta higienização das hortaliças envolve as etapas de seleção retirando as folhas, partes e unidades deterioradas, lavagem individual de cada folha em água corrente, e tratamento com água clorada 200 ppm através da submersão do material foliar por 10 minutos (34).

\section{CONCLUSÕES}

Foi constatada a presença de larvas de vida livre e protozoários ciliados e/ou flagelados, em todas as amostras analisadas, os quais em grande parte das situações não representam impacto sobre a saúde humana.

Porém, em $22,5 \%$ das amostras foram encontradas contaminações por trofozoítos e/ou cistos de ameba, fato que merece uma maior atenção pelos órgãos públicos responsáveis pela fiscalização, uma vez que vegetais consumidos in natura insuficientemente higienizados podem ser potenciais transmissores de doenças.

Desse modo, a atuação da Vigilância Sanitária nos locais de produção e distribuição de hortaliças, assim como adequadas condições higiênico-sanitárias, são indispensáveis para a obtenção de um produto de boa qualidade.

\section{REFERÊNCIAS}

1. WHO. World Health Organization. Working to overcome the global impact ofneglected tropical diseases: First WHO report on neglected tropical diseases. France; 2010.

2. Hotez PJ, Bottazzi MH, Franco-Paredes C, Ault SK, Periago MR. The neglected tropical diseases of Latin America and the Caribbean: review of estimated disease burden and distribution and a roadmap for control and elimination. PLoS Negl Trop Dis. 2008. 2(9): e300. DOI: 10.1371/journal.pntd.0000300

3. Lindoso JA, Lindoso AABP. Neglected tropical diseases in Brazil. Rev. Inst. Med. Trop. S. Paulo. 2009. 51(5): 247-253. DOI: $10.1590 / \mathrm{S} 0036-46652009000500003$

4. Soares B, Cantos GA. Qualidade parasitológica e condições higiênico-sanitárias de hortaliças comercializadas na cidade de Florianópolis, Santa Catarina, Brasil. Rev Bras Epidemiol. 2005. 8(4): 377-384. DOI:10.1590/S1415$-790 \times 2005000400006$

5. Demartini AP, Grazziotin NA. Avaliação de enteroparasitas, patogênicos ao homem em alfaces (Lactuca sativa) comercializadas na cidade de Erechim/RS. Revista Perspectiva. 2009. 33(121): 20-38.

6. Mascarello Junior IJ, Grazziotin NA, Jaskulski MR. Avaliação microbiológica de alface (Lactuca sativa) comercializada em feiras livres da cidade de Erechim, RS. Hig. Aliment. 2013. 27(220/221); 147-151.
7. Montanher CC, Coradin DC, Silva SEF. Avaliação parasitológica em alfaces (Lactuca sativa) comercializadas em restaurantes self-service por quilo, da cidade de Curitiba, Paraná, Brasil. Estud Biol. 2007. 29(6): 63-71.

8. BRASIL. Ministério da Saúde. Secretaria de Atenção a Saúde. Guia alimentar para a população brasileira: promovendo a alimentação saudável. Série A. Normas e Manuais Técnicos. 1.ed. Brasília (DF); 2006.

9. Facioli VM, Grazziotin NA. Análise parasitológica de agriões comerciais na cidade Erechim, RS. Hig. aliment. 2010. 24(180/181): 128-131.

10. Santos NM, Sales EM, Santos ABM, Damasceno KA, The TS. Avaliação parasitológica de hortaliças comercializadas em supermercados e feiras livres no município de Salvador/Ba. R. Ci. méd. biol, 2009. 8(2): 46-152.

11. Falavigna, DLM, Falavigna AL, Pupulim ART, Dias MLGG, Nishi L, Melo GC. Análise parasitológica de hortaliças comercializadas na central de abastecimento do município de Maringá-Paraná. Salusvita. 2006. 25(3): 43-51.

12. Bonilla-Lemus R, Villegas ASC, Jiménez JC, Vázquez AL. Occurrence of free-living amoebae in streams of the Mexico Basin. Experimental Parasitology. 2014. 145: 2833 DOI: 10.1016/j.exppara.2014.07.001 
13. Vargas-Zepeda J, Gómez-Alcalá AV, Vázquez-Morales JA, Licea-Amaya L, Jonckheere JF, Lares-Villa F. Successful Treatment of Naegleria fowleri Meningoencephalitis by using Intravenous Amphotericin B, Fluconazole and Rifampicin. Arch Med Res. 2005. 36(1): 83-86. DOI: 10.1016/j.arcmed.2004.11.003

14. Pimentel LA, Dantas AFM, Uzal F, Riet-Corrrea F. Meningoencephalitis caused by Naegleria fowleri in cattle of northeast Brazil. Res Vet Sci. 2012. 93(2): 811-812. DOI: 10.1016/j.rvsc.2012.01.002

15. Esteves FAM, Figueirôa EO. Detecção de enteroparasitas em hortaliças comercializadas em feiras livres do município de Caruaru (PE). RBSP. 33(2): 184-193.

16. Hotez PJ, Brindley PJ, Bethony JM, King CH, Pearce EJ, Jacobson J. Helminth infections: the great neglected tropical diseases. J Clin Invest. 2008. 118(4): 1311-1321. DOI: 10.1172/JCI34261

17. Castineiras TMPP, Martins FSV. Infecções por helmintos e enteroprotozoários. Centro de Informações Saúde Pública. CIVES-UFRJ, 2000-2002.

18. Andrade EC, Leite ICG, Rodrigues VO, Goldner CM. Intestinal parasitic diseases: a review of social, epidemiologic, clinical and therapeutic aspects. Rev APS. 2010. 13(2): 231-240, 2010.

19. Chaves ACP, Seixas Filho JT, Dantas MML. Revisão do mecanismo fisiopatológico da amebíase. Revista Augustus. 2010. 14(29): 74-87.

20. Rodriguez-Zaragoza S. Ecology of Free-Living Amoebae. Crit Rev Microbiol. 1994. 20(3): 225-24. DOI:10.3109/10408419409114556

21. Silva MA, Rosa JA. Isolation of potencially pathogenic free-living amoebas in hospital dust. Rev Saúde Pública. 2003. 37(2): 242-246. DOI: 10.1590/S003489102003000200013

22. Teixeira LH, Rocha S, Pinto RM, Caseiro MM, Costa SO. Prevalence of potentially pathogenic free-living amoebae from Acanthamoeba and Naegleria genera in non-hospital, public, internal environments from the city of Santos, Brazil. BJID. 2009. 13(6): 395-397. DOI: 10.1590/ S1413-86702009000600001.

23. Scheikl U, Sommer R, Kirschner A, Rameder A, Schrammel B, Zweimüller Z, Wesner W, Hinker M, Walochnik J. Free-living amoebae (FLA) co-occurring with legionellae in industrial Waters. Parasitology. 2014. 50(4): 422-429; DOI: $10.1016 /$ j.ejop.2014.04.002
24. Magnet A, Fenoy S, Galván AL, Izquierdo F, Rueda C, Fernandez Vadillo C, Del Aguila C. A year long study of the presence of free living amoeba in Spain. Water Res. 2013. 47(19): 6966-6972. DOI: 10.1016/j.watres.2013.09.065

25. Jonckheere JF. Origin and evolution of the worldwide distributed pathogenic amoeboflagellate Naegleria fowleri. Infect Genet Evol. 2011. 11(7): 1520-1528. DOI: 10.1016/j.meegid.2011.07.023.

26. Heggie, TW. Swimming with death: Naegleria fowleri infections in recreational Waters. Travel Med Infect Dis. 2010. 8(4):201-206. DOI:10.1016/j.tmaid.2010.06.001

27. Knickelbein JE, Kovarik J, Dhaliwal DK, Chu CT. Acanthamoeba keratitis: A clinicopathologic case report and review of the literature. Hum Pathol. 2013. 44(5): 918922. DOI: 10.1016/j.humpath.2012.10.007

28. Greub G, Raoult D. Microorganisms resistant to free-living amoebae. Clin Microbiol Rev. 2004. 17(2): 413-433. DOI: 10.1128/CMR.17.2.413-433.2004

29. Cateau E, Delafont V, Hechard Y, Rodier MH. Free-living amoebae: what part do they play in healthcare-associated infections? J Hosp Infect. 2014. 87(3): 131-140. DOI: 10.1016/j.jhin.2014.05.001

30. Souza FR. Avaliação da contaminação parasitária das hortaliças in natura comercializadas em feiras livres e supermercados da cidade de Jataí-GO. In: Congresso de Pesquisa, Ensino e Extensão da UFG. 2006. Goiânia: Anais eletrônicos do XIV Seminário de Iniciação Científica; 2006.

31. Falavigna DLM, Freitas CBR, Melo GC, Nishi L, Araujo LM, Falavigna-Guilherme AL. Qualidade de hortaliças comercializadas no noroeste do Paraná, Brasil. Parasitol Latinoam. 2005. 60(3-4): 144-149.

32. Silva MS. Avaliação parasitológica de cinco horticulturas de Nova Xavantina, MT. [Trabalho de Conclusão de Curso]. Nova Xavantina: Universidade do Estado de Mato Grosso, Campus de Nova Xavantina. 2004.

33. Soares B, Cantos GA. Detecção de estruturas parasitárias em hortaliças comercializadas na cidade de Florianópolis, SC, Brasil. Rev Bras Cienc Farm. 2006. 42(3): 455-460. DOI: $10.1590 / \mathrm{S} 1516-93322006000300015$

34. BRASIL. Agência Nacional de Vigilância Sanitária. Resolução no 216/2004. Cartilha sobre Boas Práticas para serviços de alimentação. Brasília (DF); 2006. 